Tôhoku Math. Journ.

31 (1979), 469-489.

\title{
HOLOMORPHIC FAMILIES OF RIEMANN SURFACES AND TEICHMÜLLER SPACES II
}

\author{
Applications to the uniformization of algebraic surfaces and the \\ compactification of two dimensional Stein manifolds
}

YoICHI IMAYOSHI

(Received July 19, 1978, revised February 23, 1979)

Introduction. Let $\bar{S}$ be a two dimensional complex manifold and let $C$ be a non-singular one dimensional analytic subset of $\overline{\mathscr{S}}$ or an empty set. Denote by $D$ the unit disc $|t|<1$ and by $D^{*}$ the punctured unit disc $0<|t|<1$ in the complex $t$-plane. We assume that a proper holomorphic mapping $\bar{\pi}: \overline{\mathscr{S}} \rightarrow D^{*}$ satisfies the following two conditions;

1) $\bar{\pi}$ is of maximal rank at every point of $\overline{\mathscr{S}}$, and

2) by setting $\mathscr{S}=\bar{S}-C$ and $\pi=\bar{\pi} \mid \mathscr{S}$, the fibre $S_{t}=\pi^{-1}(t)$ of $\mathscr{S}$ over each $t \in D^{*}$ is an irreducible analytic subset of $\mathscr{S}$ and is of fixed finite type $(g, n)$ with $2 g-2+n>0$ as a Riemann surface, where $g$ is the genus of $S_{t}$ and $n$ is the number of punctures of $S_{t}$. We call such a triple $\left(\mathscr{S}, \pi, D^{*}\right)$ a holomorphic family of Riemann surfaces of type $(g, n)$ over $D^{*}$. We also say that $\mathscr{S}$ has a holomorphic fibration $\left(\mathscr{S}, \pi, D^{*}\right)$ of type $(g, n)$.

Our main problem is to construct a completion of $\left(\mathscr{S}, \pi, D^{*}\right)$ canonically in such a way that the central fibre is a Riemann surface (possibly with nodes) of the same type $(g, n)$ modulo a finite group of automorphisms.

As a continuation of the preceeding paper [6], we treat the completion of $\left(\mathscr{S}, \pi, D^{*}\right)$ in the first half of this paper. For a holomorphic family $\left(\mathscr{S}, \pi, D^{*}\right)$ of Riemann surfaces of type $(g, n)$ with $2 g-2+n>0$, we regard the fibre $S_{t}$ over $t \in D^{*}$ as a point $\Phi(t)$ in a Teichmüller space. It should be noted that, in general, $\Phi$ is a multi-valued analytic mapping. In $\S 1$ and $\S 2$, we recall terminologies and notations in [6]. In $\S 3$, we study the behavior of $\Phi$ as $t$ tends to zero. In $\S 4$, using the result of $\S 3$, we canonically construct a completion $(\hat{\mathscr{S}}, \hat{\pi}, D)$ of $\left(\mathscr{S}, \pi, D^{*}\right)$ and, in $\S 5$, we prove an extension theorem for a holomorphic mapping $F$ of $\mathscr{S}$ into $\hat{\mathscr{S}}$ with $\pi=\hat{\pi} \circ F$.

In the second half of this paper, as applications of the above results, 
in $\S 6$, we deal with a uniformization theorem of two dimensional projective algebraic manifolds, which supplements Griffiths's uniformization theorem in [5]. In $\S 7$, we study a compactification of two dimensional Stein manifolds with holomorphic fibration of type $(g, n)$ with $2 g-2+n>$ 0 over finite Riemann surfaces and discuss a condition for an analytic automorphism of $C^{2}$ to be a polynomial map.

The author would like to express his hearty gratitude to Professor Tadashi Kuroda for his constant encouragement and advice.

1. Terminologies and notations. 1 . Let $G$ be a finitely generated Fuchsian group, acting on the upper half-plane $U$, of the first kind with no elliptic elements such that the quotient space $S=U / G$ is a finite Riemann surface of type $(g, n)$ with $2 g-2+n>0$. Denote by $Q_{\text {norm }}(G)$ the set of all quasiconformal automorphisms $w$ of $U$ such that $w(0)=0$, $w(1)=1, w(\infty)=\infty$ and $w G w^{-1} \subset S L^{\prime}(2 ; \boldsymbol{R})$, where $S L^{\prime}(2 ; \boldsymbol{R})$ is the set of all real Möbius transformations. Two elements $w_{1}$ and $w_{2}$ of $Q_{\text {norm }}(G)$ are called equivalent if $w_{1}=w_{2}$ on the real axis. The Teichmüller space $T(G)$ of $G$ is the set of all equivalence classes obtained by classifing $Q_{\text {norm }}(G)$ by the above equivalence relation. We denote by $[w]$ the equivalence class represented by an element $w$ of $Q_{\text {norm }}(G)$. Let $B_{2}(L, G)$ be the space of all bounded holomorphic quadratic differentials for $G$ on the lower half-plane $L$, that is, the set of all holomorphic functions $\phi$ on $L$ such that

$$
\phi(g(z)) g^{\prime}(z)^{2}=\phi(z)
$$

for every $z$ of $L$ and for every $g$ of $G$, and such that its norm

$$
\|\dot{\phi}\|=\sup _{z \in L}(\operatorname{Im} z)^{2}|\phi(z)|
$$

is finite. This space $B_{2}(L, G)$ is a $(3 g-3+n)$ dimensional complex vector space. For any element $w_{\mu}$ of $Q_{\text {norm }}(G)$ with a Beltrami coefficient $\mu$ on $U$, there is a unique quasiconformal automorphism $w^{\mu}$ of the Riemann sphere $\hat{\boldsymbol{C}}$ with $w(0)=0, w(1)=1, w(\infty)=\infty$ such that $w^{\mu}$ has the Beltrami coefficient $\mu$ on $U$ and is conformal on $L$. Denote by $\phi_{\mu}$ the Schwarzian derivative of $w^{\mu}$ on $L$. Then the Teichmüller space $T(G)$ is canonically identified with a holomorphically convex bounded domain of $B_{2}(L, G)$ by the mapping sending $\left[w_{\mu}\right]$ into $\phi_{\mu}$. We associate with every $\phi$ of $B_{2}(L, G)$ a uniquely determined solution $W_{\phi}(z)=\eta_{1}(z) / \eta_{2}(z)$ of the Schwarzian differential equation on $L$

$$
\left(w^{\prime \prime} / w^{\prime}\right)^{\prime}-\frac{1}{2}\left(w^{\prime \prime} / w^{\prime}\right)^{2}=\phi,
$$


where $\eta_{1}$ and $\eta_{2}$ are solutions of the linear differential equation on $L$

$$
2 \eta^{\prime \prime}(z)+\phi(z) \eta(z)=0
$$

normalized by the conditions $\eta_{1}=\eta_{2}^{\prime}=1$ and $\eta_{1}^{\prime}=\eta_{2}=0$ at $z=-i$. For every point $\phi$ of $T(G)$, the mapping $W_{\phi}$ defined as above is conformal on $L$ and has a quasiconformal extension of $\hat{\boldsymbol{C}}$ onto itself, which is denoted by the same notation. If we set $G_{\phi}=W_{\phi} \circ G \circ W_{\phi}^{-1}$ and $D_{\phi}=W_{\phi}(U)$, then $G_{\phi}$ is a quasi-Fuchsian group and the definitions are legitimate since $D_{\phi}$ is the complement of the closure of $W_{\phi}(L)$ and since $W_{\phi} \mid L$ depends only on $\phi$. Koebe's one-quarter theorem shows $D_{\phi} \subset(|w|<2)$ for every $\phi$ of $T(G)$.

2. Let $\left(\mathscr{S}, \pi, D^{*}\right)$ be a holomorphic family of Riemann surfaces of type $(g, n)$ with $2 g-2+n>0$. For a fixed point $t_{0}$ of $D^{*}$, let $\psi$ be a quadratic differential in $T(G)$ such that the quotient space $D_{\psi} / G_{\psi}$ is conformally equivalent to the fibre $S_{t_{0}}$ of $\mathscr{S}$ over $t_{0}$. In a sufficiently small neighborhood $\delta$ of $t_{0}$ in $D^{*}$, there is a uniquely determined holomorphic mapping $\Phi_{0}$ of $\delta$ into $T(G)$ with $\Phi_{0}\left(t_{0}\right)=\psi$ such that $D_{\Phi_{0}(t)} / G_{\Phi_{0}(t)}$ is conformally equivalent to $S_{t}$ for every $t$ of $\delta$. Moreover, $\Phi_{0}$ can be continued analytically along every path in $D^{*}$. Thus we have an analytic mapping $\Phi$ of $D^{*}$ into $T(G)$, but this is not necessarily single-valued.

Let $\widetilde{D}$ be the unit disc $|\tau|<1$ in the complex $\tau$-plane. We regard $\widetilde{D}$ as a universal covering space of $D^{*}$ with the covering map $p(\tau)=$ $\exp [2 \pi(\tau+1) /(\tau-1)]$, whose covering transformation group is generated by the transformation

$$
\gamma(\tau)=\frac{(1-2 i) \tau-1}{\tau-(1+2 i)}
$$

Take a point $\tau_{0}$ of $D$ with $p\left(\tau_{0}\right)=t_{0}$. Since $\widetilde{D}$ is simply connected, there is a single-valued analytic mapping $\widetilde{\Phi}$ of $\widetilde{D}$ into $T(G)$ with $\widetilde{\Phi}\left(\tau_{0}\right)=\psi$ such that the quotient space $D_{\widetilde{\Phi}(\tau)} / G_{\widetilde{\Phi}(\tau)}$ is conformally equivalent to $S_{p(\tau)}$ for every $\tau$ of $\widetilde{D}$. We call $\widetilde{\Phi}$ a representation of $\left(\mathscr{S}, \pi, D^{*}\right)$ into $T(G)$. Moreover, there exists an element $\mathscr{C}$ of the modular $\operatorname{group} \operatorname{Mod}(G)$ of $G$ with $\widetilde{\Phi}(\gamma(\tau))=\mathscr{M}(\widetilde{\Phi}(\tau))$ for every $\tau$ of $\widetilde{D}$. This element $\mathscr{M}$ is called the homotopic monodromy of $\left(\mathscr{S}, \pi, D^{*}\right)$ with respect to $\widetilde{\Phi}$.

3. Let $\left(S, f, S^{\prime}\right)$ be a marked Riemann surface, that is, $S=U / G$ is as before, $S^{\prime}$ is an arbitrary finite Riemann surface of type $(g, n)$ and $f$ is a quasiconformal mapping of $S$ onto $S^{\prime}$. Two marked Riemann surfaces $\left(S, f, S^{\prime}\right)$ and $\left(S, g, S^{\prime \prime}\right)$ are said to be equivalent if there exists a conformal mapping $h$ of $S^{\prime}$ onto $S^{\prime \prime}$ such that $g^{-1} \circ h \circ f$ is homotopic to 
the identity mapping of $S$. By using this equivalence relation, we classify all the marked Riemann surfaces $\left(S, f, S^{\prime}\right)$ for a given $S$ and we denote by $\left[S, f, S^{\prime}\right]$ the equivalence class represented by a marked Riemann surface $\left(S, f, S^{\prime}\right)$. We call the space $T(S)$ of all equivalence classes $\left[S, f, S^{\prime}\right]$ the Teichmüller space of $S$. For each element $\left[S, f, S^{\prime}\right]$ of $T(S)$, we can uniquely determine the element $[w]$ of $T(G)$ such that a quasiconformal mapping $w$ of $Q_{\text {norm }}(G)$ gives the following commutative diagram:

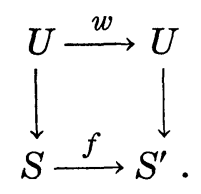

Thus the point $\left[S, f, S^{\prime}\right]$ of $T(S)$ uniquely determines a quadratic differential $\phi$ of $T(G)$. This mapping sending $\left[S, f, S^{\prime}\right]$ into $\phi$ is a biholomorphic mapping of $T(S)$ onto $T(G)$. Therefore, we may identify $T(S)$ with $T(G)$.

2. Deformation spaces of Riemann surfaces with nodes. 1 . In order to discuss the behavior of $\widetilde{\Phi}(\tau)$ as $\tau$ tends to 1 , we need the deformation spaces and moduli spaces of Riemann surfaces with nodes and punctures. In this section, we briefly explain them along Bers's line. (See [2] and [3].)

A Riemann surface $S^{\prime}$ with nodes is a connected complex space, every point $P$ of which has either a fundamental system of neighborhoods isomorphic to the unit disc $\{z \in C \quad|\quad| z \mid<1\}$ or a fundamental system of neighborhoods isomorphic to the set $\left\{\left(z_{1}, z_{2}\right) \in C^{2} \mid z_{1} z_{2}=0\right.$, $\left|z_{1}\right|<1$ and $\left.\left|z_{2}\right|<1\right\}$. In the latter case, $P$ is called a node. Each component of the complement of the set of nodes is called a part of $S^{\prime}$ and $S^{\prime}$ is called stable if every part of $S^{\prime}$ has the upper half-plane as its universal covering space.

In the following, by a Riemann surface $S^{\prime}$ of finite type we mean a stable Riemann surface with or without nodes such that $S^{\prime}$ is compact except for $n$ punctures, where $n$ is a non-negative integer. A puncture can never be at a node. In this case, $S^{\prime}$ has finitely many parts $\Sigma_{1}, \cdots, \Sigma_{r}$, and each part $\Sigma_{j}$ is of genus $g_{j}$ and is compact except for $n_{j}$ punctures with $2 g_{j}-2+n_{j}>0$ and $\sum_{j=1}^{r} n_{j}=2 k+n$, where $k$ is the number of nodes of $S^{\prime}$ and $n$ is the number of punctures of $S^{\prime}$. The total Poincaré area of $S^{\prime}$ equals $A=2 \pi \sum_{j=1}^{r}\left(2 g_{j}-2+n_{j}\right)$. The genus $g$ of $S^{\prime}$ is defined by the relation $A=2 \pi(2 g-2+n)$. The pair $(g, n)$ is called the type of $S^{\prime}$.

2. Let $S_{0}^{\prime}$ and $S^{\prime}$ be two Riemann surfaces of the same type $(g, n)$. 
A continuous surjection $\alpha: S^{\prime} \rightarrow S_{0}^{\prime}$ is called a deformation if the inverse image of every node of $S_{0}^{\prime}$ is either a node of $S^{\prime}$ or a Jordan curve on a part of $S^{\prime}$, if, for every part $\Sigma$ of $S_{0}^{\prime}$, the restriction $\alpha^{-1} \mid \Sigma$ is an orientation-preserving homeomorphism of $\Sigma$ onto $\alpha^{-1}(\Sigma)$, and if every puncture of $S^{\prime}$ corresponds to a puncture of $S_{0}^{\prime}$ under $\alpha$.

Once and for all we choose an integer $\nu(>3)$ which will be fixed throughout the following discussion.

Two deformations $\alpha: S^{\prime} \rightarrow S_{0}^{\prime}$ and $\beta: S^{\prime \prime} \rightarrow S_{0}^{\prime}$ are called equivalent to each other if there exists a homeomorphism $f$ of $S^{\prime}$ onto $S^{\prime \prime}$ with $\alpha=$ $\beta \circ f$ such that $f$ is homotopic to a product of $\nu$-th powers of Dehn twists about Jordan curves on $S^{\prime}$ mapped by $\alpha$ into nodes, followed by an isomorphism. Denote by $\langle\alpha\rangle=\left\langle S^{\prime}, \alpha, S_{0}^{\prime}\right\rangle$ the equivalence class determined by a deformation $\alpha: S^{\prime} \rightarrow S_{0}^{\prime}$. For a given Riemann surface $S_{0}^{\prime}$ of type $(g, n)$, the deformation space $X\left(S_{0}^{\prime}\right)$ is the set of equivalence classes $\langle\alpha\rangle$ obtained from all deformations $\alpha: S^{\prime} \rightarrow S_{0}^{\prime}$.

3 . In order to reduce the case $n>0$ to the case $n=0$, we shall associate with every Riemann surface $S^{\prime}$ of type $(g, n)$ a Riemann surface $a\left(S^{\prime}\right)$ of type $\left(g+g_{0}, 0\right)$, that is, a compact Riemann surface of genus $g+g_{0}$ with nodes, where $g_{0}=(4 g+8) n$. This is accomplished by attaching to each puncture $P_{j}$ on $S^{\prime}$ a "tagging" Riemann surface $V_{j}$. For a deformation $\alpha: S^{\prime} \rightarrow S_{0}^{\prime}$, we denote by $a(\alpha)$ the unique deformation of $a\left(S^{\prime}\right)$ onto $a\left(S_{0}^{\prime}\right)$ such that $a(\alpha) \mid S^{\prime}=\alpha$ and that $a(\alpha) \mid V_{j}$ is an isomorphism for $j=1, \cdots, n$. We observe $a(\mathrm{id})=\mathrm{id}$ and $a(\alpha \circ \beta)=a(\alpha) \circ a(\beta)$. Furthermore, we see for a fixed type $(g, n)$ that two Riemann surfaces $a\left(S^{\prime}\right)$ and $a\left(S^{\prime \prime}\right)$ are isomorphic if and only if $S^{\prime}$ and $S^{\prime \prime}$ are isomorphic and that two deformations $a(\alpha)$ and $a(\beta)$ are equivalent if and only if $\alpha$ and $\beta$ are equivalent. Every deformation $a\left(S^{\prime}\right) \rightarrow a\left(S_{0}^{\prime}\right)$ is equivalent to one of the forms $a(\alpha)$. Therefore, the deformation space $X\left(S_{0}^{\prime}\right)$ of a Riemann surface $S_{0}^{\prime}$ of type $(g, n)$ is identified with the intersection of $n+k_{0}$ distinguished subsets of $X\left(a\left(S_{0}^{\prime}\right)\right)$, where $k_{0}=(12 g+22) n$ is the number of nodes on $V_{1}, \cdots, V_{n}$, and for a given deformation $g: S_{1}^{\prime} \rightarrow S_{0}^{\prime}$, the allowable mapping $g_{*}: X\left(S_{1}^{\prime}\right) \rightarrow$ $X\left(S_{0}^{\prime}\right)$ sending $\left\langle S^{\prime}, \alpha, S_{1}^{\prime}\right\rangle$ into $\left\langle S^{\prime}, g \circ \alpha, S_{0}^{\prime}\right\rangle$ is the restriction of the allowable mapping $a(g)_{*}: X\left(a\left(S_{1}^{\prime}\right)\right) \rightarrow X\left(a\left(S_{0}^{\prime}\right)\right)$. Thus we see that the case $n>0$ is reduced to the case $n=0$.

4. We assume that a Riemann surface $S_{0}^{\prime}$ of finite type $(g, n)$ has $r$ parts $\Sigma_{1}, \cdots, \Sigma_{r}$ and $k$ nodes and that each part $\Sigma_{j}$ has genus $g_{j}$ and $n_{j}$ punctures. The associated compact Riemann surface $a\left(S_{0}^{\prime}\right)$ has genus $g+g_{0}, r+r_{0}$ parts $\Sigma_{1}, \cdots, \Sigma_{r+r_{0}}$ and $k+n+k_{0}$ nodes $P_{1}, \cdots, P_{k+n+k_{0}}$, where $g_{0}=(4 g+8) n, r_{0}=(8 g+15) n$ and $k_{0}=(12 g+22) n$.

In order to construct the parametrization space $X^{\prime}\left(a\left(S_{0}^{\prime}\right)\right)$ consisting 
of all Riemann surfaces with nodes which can be deformed to a given Riemann surface $a\left(S_{0}^{\prime}\right)$, we choose $r+r_{0}$ Fuchsian groups $H_{1}, \cdots, H_{r+r_{0}}$ acting on discs $\Delta_{1}, \cdots, \Delta_{r+r_{0}}$ with disjoint closures as follows: i) Each $H_{j}, j=$ $1, \cdots, r$, has $n_{j}$ non-conjugate maximal elliptic cyclic subgroups with the same fixed order $\nu(>3)$ and, for $\Delta_{j}^{\prime}$ obtained from $\Delta_{j}$ by removing all elliptic fixed points, the Riemann surface $\Delta_{j}^{\prime} / H_{j}$ is conformally equivalent to $\Sigma_{j}$, ii) each $H_{j}, j=r+1, \cdots, r+r_{0}$, has 3 non-conjugate maximal elliptic cyclic subgroups with the same fixed order $\nu$ and the Riemann surface $\Delta_{j}^{\prime} / H_{j}$ is of type $(0,3)$ and iii) $H_{1}, \cdots, H_{r+r_{0}}$ generate a Kleinian group $H$ which is their product and has an invariant component $\Delta_{0}$.

We assign to each node $P_{i}$ of $a\left(S_{0}^{\prime}\right)$ two non-conjugate maximal elliptic cyclic subgroups $\Gamma_{i}^{\prime}, \Gamma_{i}^{\prime \prime}$ of $H$ so that, if $P_{i}$ joins $\Sigma_{j}$ to $\Sigma_{l}$, then $\Gamma_{i}^{\prime} \subset H_{j}$ and $\Gamma_{i}^{\prime \prime} \subset H_{l}$. Two elliptic fixed points not contained in $\Delta_{0}$ are called related if they are fixed under elliptic cyclic subgroups conjugate to either $\Gamma_{i}^{\prime}$ or $\Gamma_{i}^{\prime \prime}$. These $\Gamma_{i}$ are chosen in such a way that the union of $\Delta_{j} / H_{j}$, with the images of any two related elliptic fixed points identified, is isomorphic to $a\left(S_{0}^{\prime}\right)$.

A point $\left\langle S^{\prime}, \alpha, S_{0}^{\prime}\right\rangle$ in $X\left(S_{0}^{\prime}\right)$ is represented by a point $(\xi, \eta)=\left(\xi_{1}, \cdots\right.$, $\left.\xi_{r}, \eta_{1}, \cdots, \eta_{k}, 0, \cdots, 0\right)$ in $X^{\prime}\left(a\left(S_{0}^{\prime}\right)\right)$, where every $\xi_{j}, j=1, \cdots, r$, is a point in the Teichmüller space $T\left(H_{j}\right)$ and every $\eta_{i}, i=1, \cdots, k$, is a complex number. Let $H(\xi, \eta)$ be the Kleinian group determined by the point $(\xi, \eta)$ in $X^{\prime}\left(a\left(S_{0}^{\prime}\right)\right)$. Denote by $\Omega(\xi, \eta)$ the part of the region of discontinuity of $H(\xi, \eta)$ corresponding to $S^{\prime}$ and denote by $\Omega^{\prime}(\xi, \eta)$ the complement, in $\Omega(\xi, \eta)$, of all elliptic fixed points. Then the quotient space $\Omega(\xi, \eta) / H(\xi, \eta)$, with the images of any two related elliptic fixed points identified, is a Riemann surface $S_{\xi, \eta}$ with nodes of type $(g, n)$ and is isomorphic to $S^{\prime}$. This surface $S_{\xi, \eta}$ is equipped with a canonical deformation $\alpha_{\xi, \eta}$ with $\left\langle S_{\xi, \eta}, \alpha_{\xi, \eta}, S_{0}^{\prime}\right\rangle=\left\langle S^{\prime}, \alpha, S_{0}^{\prime}\right\rangle$, up to equivalence.

3. Holomorphic families of Riemann surfaces. Riemann's moduli space $R(g, n)$ is the set of isomorphism classes [ $\left[S^{\prime}\right]$ of all Riemann surfaces $S^{\prime}$ without nodes and with signature $(g, n ; \infty, \cdots, \infty)$. The moduli space $M(g, n)$ is the set of isomorphism classes $\left[S^{\prime}\right]$ of all Riemmann surfaces $S^{\prime}$ with nodes of signature $(g, n ; \infty, \cdots, \infty)$. We recall the fact stated in $\S 2$ and $\S 3$ that the case $n>0$ can be reduced to the case $n=0$. Then we see that $R(g, n)$ can be regarded as a Zariski-open subset of $M(g, n)$.

For a holomorphic family $\left(\mathscr{S}, \pi, D^{*}\right)$ of Riemann surfaces of type $(g, n)$ with $2 g-2+n>0$, the holomorphic mapping $J: D^{*} \rightarrow R(g, n)$ sending $t$ into $\left[S_{t}\right]$ has a holomorphic extension $\hat{J}: D \rightarrow M(g, n)$. This fact can be proved by a reasoning similar to that in the proof of Lemma 1 
in [6].

Now let $S=U / G$ be the fixed Riemann surface as before. If we set $\widehat{J}(0)=\left[S_{0}\right]$ for a Riemann surface $S_{0}$ with or without nodes of signature $(g, n ; \infty, \cdots, \infty)$, then there exists a deformation $\alpha: S \rightarrow S_{0}$ such that $\left\langle S_{\tau}, \alpha \circ f_{\tau}^{-1}, S_{0}\right\rangle$ converges uniformly to $\langle\mathrm{id}\rangle$ in $X\left(S_{0}\right)$ as $\tau$ tends to 1 through any cusp region $\Delta$ at $\tau=1$ in $\widetilde{D}$, where $\left[S, f_{\tau}, S_{\tau}\right]$ is the point of the Teichmüller space $T(S)$ of $S$ and corresponds to the point $\widetilde{\Phi}(\tau)$ in $T(G)$.

Thus we obtain the following theorem, whose proof is similar to that of Theorem 1 in [6] and may be omitted.

TheORem 1. Let $\left(\mathscr{S}, \pi, D^{*}\right)$ be a holomorphic family of Riemann surfaces of type $(g, n)$ with $2 g-2+n>0$. Then there is an element $\phi_{1}$ in the closure of $T(G)$ in $B_{2}(L, G)$ such that a representation $\widetilde{\Phi}(\tau)$ of $\left(\mathscr{S}, \pi, D^{*}\right)$ into $T(G)$ converges to $\phi_{1}$ uniformly as $\tau$ tends to 1 through any cusp region $\Delta$ at $\tau=1$ in $\widetilde{D}$. The homotopic monodromy $\mathscr{C}$ of $\left(\mathscr{S}, \pi, D^{*}\right)$ with respect to $\widetilde{\Phi}$ is of finite order if and only if $\phi_{1} \in T(G)$, and is of infinite order if and only if $\phi_{1} \in \partial T(G)$, where $\partial T(G)$ is the boundary of $T(G)$ in $B_{2}(L, G)$. In the latter case, the boundary group $G_{1}$ corresponding to $\phi_{1} \in \partial T(G)$ is a regular b-group.

REMARK. In Theorem 1, let $D_{1}=\Omega\left(G_{1}\right)-\Delta\left(G_{1}\right), \quad \Omega\left(G_{1}\right)$ be the region of discontinuity of $G_{1}$ and let $\Delta\left(G_{1}\right)$ be the invariant component of $G_{1}$. Let $S_{0}$ be a Riemann surface with nodes of signature $(g, n ; \infty, \cdots, \infty)$ with $\hat{J}(0)=\left[S_{0}\right]$ obtained in the beginning of this section. Then the quotient space $D_{1} \cup$ fixed points of accidental parabolic elements of $\left.G_{1}\right\} / G_{1}$ is isomorphic to $S_{0}$.

4. Completion of holomorphic families of Riemann surfaces. 1. In order to canonically construct the completion $(\hat{\mathscr{S}}, \hat{\pi}, D)$ of a holomorphic family $\left(\mathscr{S}, \pi, D^{*}\right)$ of Riemann surfaces of type $(g, n)$ with $2 g-2+n>0$, we need some preliminaries. We use the previous notations. Let $\left[S, f_{\tau}, \widetilde{S}_{\tau}\right]$ be the point of the Teichmüller space $T(S)$ of the fixed Riemann surface $S=U / G$ corresponding to the point $\widetilde{\Phi}(\tau)$ of the Teichmüller space $T(G)$ of $G$ for each $\tau$ of $\widetilde{D}$. Let $S_{0}$ be a Riemann surface with or without nodes of signature $(g, n ; \infty, \cdots, \infty)$ obtained in $\S 3$. The point $\left\langle\widetilde{S}_{\tau}, \alpha \circ f_{\tau}^{-1}\right.$, $\left.S_{0}\right\rangle$ in $X\left(S_{0}\right)$ is represented by a point $(\xi(\tau), \eta(\tau))$ of the parametrization space $X^{\prime}\left(a\left(S_{0}\right)\right)$ for each $\tau$ of $\widetilde{D}$ and the point $(\xi(\tau), \eta(\tau))$ determines the point $\left\langle S_{\xi(\tau), \eta(\tau)}, \alpha_{\hat{\varepsilon}(\tau), \eta(\tau)}, S_{0}\right\rangle$ of $X\left(S_{0}\right)$. We denote by $\left[\Sigma_{j}, F_{j, \tau}, \Sigma_{j, \tau}\right]$ the point of the Teichmüller space $T\left(\Sigma_{j}\right)$ of $\Sigma_{j}$ corresponding to the point $\xi_{j}(\tau)$ in $T\left(H_{j}\right)$ for $j=1, \cdots, r$. Let $\widetilde{H}_{j}, j=1, \cdots, r$, be the Fuchsian group determined by the universal covering of $\Sigma_{j}$. We regard $\left[\Sigma_{j}, F_{j, z}, \Sigma_{j, r}\right]$ 
as a point $\phi_{j, \tau}$ of the Teichmüller space $T\left(\widetilde{H}_{j}\right)$ of $\widetilde{H}_{j}$. As was stated in $\S 3,\left\langle a\left(\widetilde{S}_{\tau}\right), a\left(\alpha \circ f_{\tau}^{-1}\right), a\left(S_{0}\right)\right\rangle$ converges uniformly to $\langle$ id $\rangle$ in $X\left(a\left(S_{0}\right)\right)$ as $\tau$ tends to 1 through any cusp region $\Delta$ at $\tau=1$ in $\widetilde{D}$. Hence we see that holomorphic quadratic differentials $\phi_{j, \tau}$ in $T\left(\tilde{H}_{j}\right)$ converge to zero as $\tau$ tends to 1 in $\Delta$. Since $\left\langle\widetilde{S}_{\tau}, \alpha \circ f_{\tau}^{-1}, S_{0}\right\rangle$ is equal to $\left\langle S_{\xi(\tau), \eta(\tau)}, \alpha_{\xi(\tau), \eta(\tau)}, S_{0}\right\rangle$, by definition, there exists a homeomorphism $g_{\tau}: S_{\xi(\tau), \eta(\tau)} \rightarrow \widetilde{S}_{\tau}$ with the relation $\alpha_{\xi(\tau), \eta(\tau)}=\alpha \circ f_{\tau}^{-1} \circ g_{\tau}$ such that $g_{\tau}$ is homotopic to a product $d_{\tau}$ of $\nu$-th powers of Dehn twists about Jordan curves mapped by $\alpha_{\xi(\tau), \eta(\tau)}$ into nodes, followed by an isomorphism. Thus we see that $\left[S, f_{\tau}, \widetilde{S}_{\tau}\right]=\left[S, d_{\tau} \circ g_{\tau}^{-1} \circ f_{\tau}\right.$, $\left.S_{\xi(\tau), \eta(\tau)]}\right]$ in $T(S)$.

Let $P_{1}, \cdots, P_{k}$ be the nodes of $S_{0}$ and let $\delta_{j}$ be a sufficiently small neighborhood of each $P_{j}$, and let $\delta=\delta_{1} \cup \cdots \cup \delta_{k}$. We may assume that the deformations $\alpha$ and $\alpha_{\xi(\tau), \eta(\tau)}$ are locally quasiconformal mappings on $S-\bigcup_{j=1}^{k} \alpha^{-1}\left(P_{j}\right)$ and $S_{\xi(\tau), \eta(\tau)}-\bigcup_{j=1}^{k} \alpha_{\xi(\tau), \eta(\tau)}^{-1}\left(P_{j}\right)$, respectively. There exists a quasiconformal mapping $h_{\delta, \tau}: S \rightarrow S_{\xi(\tau), \eta(\tau)}$ with $\left[S, f_{\tau}, \widetilde{S}_{\tau}\right]=$ $\left[S, h_{\hat{\delta}, \tau}, S_{\xi(\tau), \eta(\tau)}\right]$ and with $h_{\hat{\delta}, \tau}=d_{\tau} \circ g_{\tau}^{-1} \circ f_{\tau}$ on $S-\alpha^{-1}(\delta)$. Let $\pi_{0}: U \rightarrow U / G=$ $S$ and $\tilde{\pi}_{j}: U_{j} \rightarrow U_{j} / \widetilde{H}_{j}=\Sigma_{j}$ for each $j$ be the natural projections, where $U_{j}$ is the upper half $z_{j}$-plane. Let $\Delta_{j, j}$ be a connected component of $\pi_{0}^{-1} \circ \alpha^{-1}\left(\Sigma_{j}-\delta\right)$ and let $\widetilde{\Delta}_{\delta, j}=\pi_{j}^{-1}\left(\Sigma_{j}-\delta\right)$. We can lift $\alpha$ to a quasiconformal mapping $A_{j}: \Delta_{\delta, j} \rightarrow \widetilde{\Delta}_{\delta, j}$ such that $A_{j}$ conjugates the subgroup $G_{j}^{\prime}$ of $G$ corresponding to the fundamental group of $S_{j}^{\prime}=\alpha^{-1}\left(\Sigma_{j}\right)$ into $\widetilde{H}_{j}$.

Let $W_{\delta, \tau}=W_{\phi_{\tau}}$ be the quasiconformal automorphism of $\hat{\boldsymbol{C}}$ defined in $\S 1$ by $\phi_{\tau}=\left[S, h_{\delta, \tau}, S_{\xi(\tau), \eta(\tau)}\right]$, which is induced by $h_{\delta, \tau}$ on the upper halfplane $U$ and is conformal on the lower half-plane $L$. By the construction, we see that $W_{\hat{\delta}, \tau}=W_{\delta^{\prime}, \tau}$ on $\Delta_{\delta, j}$ for any $\delta$ and $\delta^{\prime}$ with $\delta^{\prime} \subset \delta$. So we may abbreviate $W_{\hat{\delta}, \tau}$ to $W_{\tau}$. We also abbreviate $\Delta_{\delta, j}$ and $\widetilde{\Delta}_{\delta, j}$ to $\Delta_{j}$ and $\widetilde{\Delta}_{j}$, respectively. Similarly, let $W_{j, \tau}=W_{\phi_{j, \tau}}$ be the quasiconformal automorphism of $\hat{\boldsymbol{C}}$ defined by $\left[\Sigma_{j}, F_{j, \tau}, \Sigma_{j, \tau}\right]$, which is induced by $F_{j, \tau}$ on the upper half-plane $U_{j}$ and is conformal on the lower half-plane $L_{j}$. We may assume that $W_{j, \tau}\left(z_{j}\right)$ is continuous for $\left(\tau, z_{j}\right)$ in $\widetilde{D} \times U_{j}$. Then the mapping

$$
V_{j, \tau}=W_{\tau} \circ A_{j}^{-1} \circ W_{j, \tau}^{-1}: W_{j, \tau}\left(\widetilde{\Delta}_{j}\right) \longrightarrow W_{\tau}\left(\Delta_{j}\right)
$$

is conformal, because $W_{\tau}$ and $W_{j, \tau} \circ A_{j}$ have the same Beltrami coefficients on $\Delta_{j}$. Since $\phi_{j, \tau} \rightarrow 0$ in $T\left(\tilde{H}_{j}\right)$ as $\tau$ tends to 1 in a cusp region $\Delta$ at $\tau=1$, we may assume that $W_{j, \tau}$ converges uniformly to the Möbius transformation $W_{j, 1}$ sending $z_{j}$ into $1 /\left(z_{j}+i\right)$ on any compact subset of $U_{j}$ as $\tau$ tends to 1 in $\Delta$. Thus, noting that $\left\{V_{j, r}\right\}_{\tau \in \Delta}$ is a normal family, we see that $\left\{W_{\tau}\right\}_{\tau \in \Delta}$ is a normal family on $U_{\delta}=U-\pi_{0}^{-1} \circ \alpha^{-1}(\delta)$ for each $\delta$. By contracting each $\delta_{j}$ to the node $P_{j}$, we can prove that $W_{\tau}$ converges uniformly on any 
compact subset of $U_{0}=U-\pi_{0}^{-1} \circ \alpha^{-1}\left(P_{1} \cup \cdots \cup P_{k}\right)$ to a locally quasiconformal mapping defined on $U_{0}$ as $\tau$ tends to 1 in $\Delta$. In fact, since $\left\{W_{\tau}\right\}_{\tau \in \Delta}$ is a normal family on $U_{\delta} \cup L$ for each $\delta$, there is a sequence $\left\{W_{\tau_{n}}\right\}_{n=1}^{\infty}$ of $\left\{W_{\tau}\right\}_{\tau \in \Delta}$ with $\tau_{n} \rightarrow 1$ as $n$ tends to $\infty$ such that $W_{\tau_{n}}$ converges uniformly on any compact subset of $U_{0} \cup L$ to a locally quasiconformal mapping $W_{1}$ defined on $U_{0} \cup L$ as $n \rightarrow \infty$. The Schwarzian derivative of $W_{1}$ on $L$ is equal to $\phi_{1}$ in Theorem 1 . Let $G_{1}$ be a quasi-Fuchsian group or a regular $b$ group corresponding to $\phi_{1}$. If $W_{1}^{\prime}$ is the limit of another sequence $\left\{W_{\tau_{n}^{\prime}}^{\prime_{n}}\right\}_{n=1}^{\infty}$ with the same properties as $\left\{W_{\tau_{n}}\right\}_{n=1}^{\infty}$, then $W_{1}^{\prime} \circ W_{1}^{-1}$ is a conformal mapping of the region of discontinuity $\Omega\left(G_{1}\right)$ of $G_{1}$ onto itself such that $W_{1}^{\prime} \circ W_{1}^{-1}$ conjugates $G_{1}$ into itself. Hence, by a theorem due to Abikoff and Marden, the mapping $W_{1}^{\prime} \circ W_{1}^{-1}$ can be extended to a conformal automorphism of $\hat{\boldsymbol{C}}$. On the other hand, by Theorem 1 , we have $W_{1}=W_{1}^{\prime}$ on $L$, which implies that $W_{1}^{\prime} \circ W_{1}^{-1}$ is the identity map of $\hat{\boldsymbol{C}}$. Thus we have $W_{1}=W_{1}^{\prime}$ on $U_{0} \cup L$. This shows that $\left\{W_{\tau}\right\}_{\tau \in \Delta}$ converges uniformly on any compact subset of $U_{0}$ to the locally quasiconformal mapping $W_{1}$ defined on $U_{0}$ as $\tau$ tends to 1 in a cusp region $\Delta$ at $\tau=1$.

2. Now, for every $\tau \in \widetilde{D}$, we set $\phi_{\tau}=\widetilde{\Phi}(\tau)$ and denote by $G_{\tau}$ the quasi-Fuchsian group corresponding to $\phi_{\tau}$. Let $\Omega\left(G_{\tau}\right)$ be the region of discontinuity of $G_{\tau}$ and let $\Delta\left(G_{\tau}\right)=W_{\phi_{\tau}}(L)$. We set $D_{\tau}=\Omega\left(G_{\tau}\right)-\Delta\left(G_{\tau}\right)$. Similarly, we set $D_{1}=\Omega\left(G_{1}\right)-\Delta\left(G_{1}\right)$, where $G_{1}=G_{\phi_{1}}$ is a quasi-Fuchsian group or a regular $b$-group corresponding to the point $\phi_{1}$ in $\overline{T(G)}$, whose existence is guranteed by Theorem 1 .

Let $\mathscr{P}_{\tau}$ be the set of all parabolic fixed points in $\partial D_{\tau}$ of $G_{\tau}$ for each $\tau$ of $\widetilde{D}$. Let $\mathscr{P}_{1}^{\prime}$ be the set of all non-accidental parabolic fixed points in $\partial D_{1}$ of $G_{1}$ and let $\mathscr{P}_{1}^{\prime \prime}$ be the set of all accidental parabolic fixed points in $\partial D_{1}$ of $G_{1}$. We set $\widetilde{D}_{\tau}=D_{\tau} \cup \mathscr{P}_{\tau}$ for $\tau \in \widetilde{D}, \mathscr{P}_{1}=\mathscr{P}_{1}^{\prime} \cup \mathscr{P}_{1}^{\prime \prime}$ and $\widetilde{D}_{1}=$ $D_{1} \cup \mathscr{P}_{1}$. Finally, we set

$$
\begin{aligned}
& \mathscr{D}=\left\{(\tau, w) \mid \tau \in \widetilde{D}, w \in D_{\tau}\right\}, \\
& \tilde{\mathscr{D}}=\left\{(\tau, w) \mid \tau \in \widetilde{D}, w \in \widetilde{D}_{\tau}\right\}, \\
& \mathscr{D}_{1}=\left\{(1, w) \mid w \in D_{1}\right\}, \\
& \tilde{\mathscr{D}}_{1}=\left\{(1, w) \mid w \in \widetilde{D}_{1}\right\}, \text { and } \\
& \hat{\mathscr{D}}=\tilde{\mathscr{D}} \cup \tilde{\mathscr{D}}_{1} .
\end{aligned}
$$

We will introduce a topology on $\hat{\mathscr{D}}$. Let us define its fundamental system of neighborhoods as follows:

i) If $a=\left(\tau_{0}, w_{0}\right)$ is in $\mathscr{D}$, then we set $z_{0}=W_{\tau_{0}}^{-1}\left(w_{0}\right)$ and take a disc $\varepsilon$ with the center $\tau_{0}$ in $\widetilde{D}$ and a disc $K$ with the center $z_{0}$ in the upper half-plane $U$. The set $\left\{(\tau, w) \mid \tau \in \varepsilon, w \in W_{\tau}(K)\right\}$ is an element of the 
fundamental system $\mathscr{L}_{a}$ of neighborhoods at $a$.

ii) If $a=\left(\tau_{0}, w_{0}\right)$ is in $\tilde{\mathscr{D}}-\mathscr{D}$, then we set $z_{0}=W_{\tau_{0}}^{-1}\left(w_{0}\right)$ and take a disc $\varepsilon$ with the center $\tau_{0}$ in $\widetilde{D}$. Take a horocycle $C$ at $z_{0}$ in $U$, that is, a Euclidean circle in $\bar{U}$ tangent to the real line at $z_{0}$. We set $K=$ (Int $C) \cup\left\{z_{0}\right\}$, where Int $C$ denotes the domain bounded by the horocycle $C$. The set $\left\{(\tau, w) \mid \tau \in \varepsilon, w \in W_{\tau}(K)\right\}$ is an element of $\mathscr{L}_{a}$.

From now on, we set $\mathscr{K}=(\operatorname{Int} \mathscr{C}) \cup\{1\}$, where $\mathscr{C}$ is a horocycle at $\tau=1$ in $\widetilde{D}$.

iii) If $a=\left(1, w_{0}\right)$ is in $\mathscr{D}_{1}$, then we set $z_{0}=W_{1}^{-1}\left(w_{0}\right)$ and take a disc $K$ with the center $z_{0}$ in $U$. The set $\left\{(\tau, w) \mid \tau \in \mathscr{K}, w \in W_{\tau}(K)\right\}$ is an element of $\mathscr{U}_{a}$.

iv) If $a=\left(1, w_{0}\right)$ with $w_{0} \in \mathscr{P}_{1}^{\prime}$, then we set $z_{0}=W_{1}^{-1}\left(w_{0}\right)$ and take a horocycle $C$ at $z_{0}$ in $U$ and set $K=(\operatorname{Int} C) \cup\left\{z_{0}\right\}$. In this case, it is observed that $W_{1}$ and $W_{1}^{-1}$ can be continuously extended to the points $z_{0}$ and $w_{0}$, respectively. The set $\left\{(\tau, w) \mid \tau \in \mathscr{K}, w \in W_{\tau}(K)\right\}$ is an element of $\mathscr{U}_{a}$.

v) If $a=\left(1, w_{0}\right)$ with $w_{0} \in \mathscr{\mathscr { P }}_{1}^{\prime \prime}$, then there exists a node $P_{0}$ on $S_{0}$ corresponding to $w_{0}$. Let $\delta_{0}$ be a sufficiently small neighborhood of $P_{0}$ in $S_{0}$ and let $K$ be the connected component of $\pi_{0}^{-1} \circ \alpha^{-1}\left(\delta_{0}\right)$ such that the closure of $W_{1}\left(K \cap U_{0}\right)$ contains the point $w_{0}$. Then the set

$$
\left\{(\tau, w) \mid \tau \in \mathscr{K}, \quad w \in W_{\tau}(K)\right\} \cup\{a\}
$$

is an element of $\mathscr{U}_{a}$.

We can prove that $\left\{\mathscr{U}_{a}\right\}_{a \in \hat{\mathscr{O}}}$ defined as above satisfies the axioms for a fundamental system of neighborhoods and induces a Hausdorff topology on $\hat{\mathscr{D}}$. In the following, we assume that $\hat{\mathscr{D}}$ is equipped with this Hausdorff topology.

3. Let $N(G)$ be the set of all quasiconformal automorphisms $\omega$ of $U$ with $\omega G \omega^{-1}=G$, and let $Q_{0}$ be the set of all quasiconformal automorphisms of $U$ which coincide with the identity on the real axis. The modular group $\operatorname{Mod}(G)$ of $G$ is defined as the factor group

$$
\operatorname{Mod}(G)=\left(N(G) /\left(N(G) \cap Q_{0}\right)\right) / G,
$$

wherc every element of $G$ is regarded as an element of $N(G)$. Every element $\langle\omega\rangle$ of $\operatorname{Mod}(G)$ defined by $\omega$ of $N(G)$ induces an automorphism of $T(G)$ sending $[w]$ into $\langle\omega\rangle([w])=\left[\omega_{*}(w)\right]$, where $w \in Q_{\text {norm }}(G)$ and $\omega_{*}(w)=$ $\lambda \circ w \circ \omega^{-1} \in Q_{\text {norm }}(G)$ with $\lambda \in S L^{\prime}(2 ; R)$.

Let $\omega_{0}$ be an element of $N(G)$ inducing the homotopic monodromy $\mathscr{C}$ of $\left(\mathscr{S}, \pi, D^{*}\right)$ with respect to the representation $\widetilde{\Phi}$, that is, $\left\langle\omega_{0}\right\rangle=\mathscr{A}$. We set $\omega=g \circ \omega_{0}^{n}$ for $g \in G$ and $n \in Z$. Let $w_{\tau} \in Q_{\text {norm }}(G)$ for $\tau \in \widetilde{D}$ be the 
quasiconformal automorphism of $U$ defined by $\left[S, h_{\hat{\delta}, r}, S_{\xi(\tau), \eta(\tau)}\right]$ as in $\S 4.1$. We set $\widetilde{w}_{\tau}=\lambda_{\tau} \circ w_{\tau} \circ \omega^{-1}$, where $\lambda_{\tau} \in S L^{\prime}(2 ; \boldsymbol{R})$ is taken in such a way that $\widetilde{w}_{\tau} \in Q_{\text {norm }}(G)$. Then $\left[\widetilde{w}_{\tau}\right]$ is identical with $\left[w_{r n_{(\varepsilon)}}\right]$ in $T(G)$, where $\gamma$ is the generator of the covering transformation group of the universal covering $p: \widetilde{D} \rightarrow D^{*}$ in $\S 1.2$. Let $W_{\tau}$ be the quasiconformal automorphism of $\hat{\boldsymbol{C}}$ which has the same Beltrami coefficient as that of $w_{\tau}$ on $U$ and which is conformal on $L$ such that

$$
W_{\tau}(z)=\frac{1}{z+i}+O(|z+i|)
$$

as $z$ tends to $z=-i$. Similarly, let $\tilde{W}_{\tau}$ be the quasiconformal automorphism of $\hat{\boldsymbol{C}}$ induced by $\widetilde{w}_{\tau}$. We set

$$
H_{\tau}(w)=\widetilde{W}_{\tau} \circ \omega \circ W_{\tau}^{-1}(w) .
$$

Then $H_{\tau}$ is a conformal bijection of $D_{\tau}$ onto $D_{\gamma^{n}(\tau)}$. Denote by $g_{n}$ the analytic automorphism of $\mathscr{D}$ sending $(\tau, w)$ into $\left(\gamma^{n}(\tau), H_{\tau}(w)\right)$. The set $\mathscr{G}=\left\{g_{n} \mid g \in G, n \in Z\right\}$ is a discrete and fixed-point-free subgroup of the analytic automorphism group of $\mathscr{D}$.

4. Here we will show that every element $g_{n}$ of $\mathscr{G}$ can be extended naturally to a homeomorphism $\hat{g}_{n}$ of $\hat{\mathscr{D}}$ onto itself. First we observe that $g_{n}$ can be extended naturally to a homeomorphism $\widetilde{g}_{n}$ of $\tilde{\mathscr{D}}$ onto itself. By the same reasoning as in $\S 4.1$, we can show that $\widetilde{W}_{\tau}$ converges uniformly on any compact subset of $\widetilde{U}_{0}=\omega\left(U_{0}\right)$ to a locally quasiconformal mapping $\widetilde{W}_{1}$ defined on $\widetilde{U}_{0}$ as $\tau$ tends to 1 through any cusp region $\Delta$ at $\tau=1$ in $\widetilde{D}$. Hence $H_{\tau}$ converges uniformly on any compact subset of $D_{1}$ to a conformal mapping $H_{1}$ of $D_{1}$ onto itself as $\tau$ tends to 1 in $\Delta$. Since $G_{1}$ is a quasi-Fuchsian group or a regular $b$-group, every component of $D_{1}$ is bounded by a quasi-circle. Therefore, by Carathéodory's theorem, the conformal mapping $H_{1}$ of $D_{1}$ onto itself can be extended to a homeomorphism $\hat{H}_{1}$ of $\bar{D}_{1}$ onto itself, where $\bar{D}_{1}$ is the closure of $D_{1}$ in $\hat{\boldsymbol{C}}$. To define the extension $\widehat{g}_{n}$ of $g_{n}$, we set $\widehat{g}_{n}=\widetilde{g}_{n}$ on $\tilde{\mathscr{D}}$ and we set $\widehat{g}_{n}(1, w)=$ $\left(1, \hat{H}_{1}(w)\right)$ on $\tilde{\mathscr{D}}_{1}$.

We shall show that $\hat{g}_{n}$ is continuous on $\hat{\mathscr{D}}$. We will prove the continuity of $\hat{g}_{n}$ at $a=\left(1, w_{0}\right) \in \grave{\mathscr{D}}_{1}-\mathscr{D}_{1}$ with $w_{0} \in \mathscr{\mathscr { P }}_{1}^{\prime \prime}$, since in the other cases, the proof is similar. We set $\hat{w}_{0}=\hat{H}_{1}\left(w_{0}\right)$ and denote by $P_{0}$ and by $\hat{P}_{0}$ the nodes of $S_{0}$ corresponding to $w_{0}$ and $\hat{w}_{0}$, respectively. Assume that $\widehat{g}_{n}$ is not continuous at the point $a$. Then we can choose an infinite sequence $\left\{\tau_{j}\right\}_{j=1}^{\infty}$ of points of $\widetilde{D}$ with $p\left(\tau_{j}\right) \rightarrow 0$ as $j$ tends to $\infty$, a neighborhood $\delta$ of $\hat{P}_{0}$ in $S_{0}$ and an infinite sequence $\left\{\delta_{j}\right\}_{j=1}^{\infty}$ of neighborhoods of $P_{0}$ in $S_{0}$ with $\delta_{j} \supset \delta_{j+1}$ for each $j$ and $\bigcap_{j=1}^{\infty} \delta_{j}=\left\{P_{0}\right\}$ 
such that, if $K$ is the connected component of $\pi_{0}^{-1} \circ \alpha^{-1}(\delta)$ with the property $\hat{w}_{0} \in \overline{W_{1}\left(K \cap U_{0}\right)}$ and if $K_{j}$ denotes the connected component of

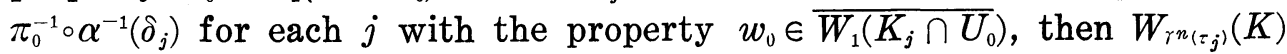
does not include $\widetilde{W}_{\tau j} \circ \omega\left(K_{j}\right)$ for each $j$, where $\omega=g \circ \omega_{0}^{n}$ is as before. Since the mapping $\hat{H}_{1}$ is continuous on $\bar{D}_{1}$, we may assume that $\hat{H}_{1}\left(W_{1}\left(K_{j} \cap U_{0}\right)\right)$ is contained in $W_{1}\left(K \cap U_{0}\right)$. By the same argument as in $\S 4.1$, we can prove that $W_{r^{n}(\bar{j})}$ converges to $W_{1}$ uniformly on any compact subset of $U_{0}$ as $j$ tends to $\infty$, and we can also prove that $\widetilde{W}_{\tau_{j}}$ converges to $\widetilde{W}_{1}$ uniformly on any compact subset of $\omega\left(U_{0}\right)$ as $j$ tends to $\infty$. Thus we may assume that $W_{r^{n}\left(\tau_{j}\right)}(K)$ intersects $\widetilde{W}_{r_{j}} \circ \omega\left(K_{j}\right)$ for each $j$. Then there are a point $\zeta_{j}$ on the boundary $\partial K$ of $K$ and a point $z_{j}$ in $K_{j}$ such that

$$
W_{r^{n}\left(\bar{z}_{j}\right)}\left(\zeta_{j}\right)=\widetilde{W}_{\tau_{j} \circ} \omega\left(z_{j}\right)=H_{\tau_{j} \circ} W_{\tau_{j}}\left(z_{j}\right) .
$$

Since $W_{\gamma^{n}\left(\tau_{j}\right)}=\widetilde{W}_{\tau_{j}}$ on $L$ and since, for the hyperbolic transformation $h$ of $G$ which makes $K_{j}$ invariant, $K$ is invariant under $h^{\prime}=\omega \circ h \circ \omega^{-1}$, we may assume that $\zeta_{j}$ converges to a point $\zeta$ in $\partial K \cap U$ as $j$ tends to $\infty$. If $\delta_{0}$ is a sufficiently small neighborhood of $\zeta$ in $U$, then $H_{\tau_{j}}^{-1} \circ W_{\left.r_{(\tau ;}\right)}$ converges to $H_{1}^{-1} \circ W_{1}$ uniformly on $\delta_{0}$ as $j$ tends to $\infty$, which implies that

$$
H_{\tau_{j}}^{-1} \circ W_{r_{\left(\tau_{j}\right)}}\left(\zeta_{j}\right)=W_{\tau_{j}}\left(z_{j}\right)
$$

converges to the point $w_{0}^{\prime}=H_{1}^{-1} \circ W_{1}(\zeta)$ in the region of discontinuity of $G_{1}$ as $j$ tends to $\infty$. On the other hand, $W_{\tau_{j}}\left(z_{j}\right)$ converges to the point $w_{0}$ of the limit set of $G_{1}$ as $j$ tends to $\infty$. Hence we have a contradiction.

As was mentioned already, the argument is similar in the other cases. Therefore, $\hat{g}_{n}$ is a homeomorphism of $\hat{\mathscr{D}}$ onto itself. Denote by $\hat{\mathscr{S}}$ the group of all such topological automorphisms $\hat{g}_{n}$ of $\hat{\mathscr{D}}$.

5. Next we introduce a normal complex structure on the quotient space $\hat{\mathscr{S}}=\hat{\mathscr{D}} / \hat{\mathscr{G}}$, which will give the completion $(\hat{\mathscr{S}}, \hat{\pi}, D)$ of $\left(\mathscr{S}, \pi, D^{*}\right)$.

Let $f_{*}$ be an element of the modular $\operatorname{group} \operatorname{Mod}(S)$ of the Teichmüller space $T(S)$ corresponding to the homotopic monodromy $/ /$ of $\left(\mathscr{S}, \pi, D^{*}\right)$. As is stated in $\S 3$, the mapping $J: D^{*} \rightarrow R(g, n)$ sending $t$ into $\left[S_{t}\right]$ has a holomorphic extension $\hat{J}: D \rightarrow M(g, n)$ with $\hat{J}(0)=\left[S_{0}\right]$. Further, as is shown by Bers [2], there exist a neighborhood $N$ of $\langle$ id $\rangle$ in $X\left(S_{0}\right)$ and the (finite) isotropy group $\Gamma_{0}\left(S_{0}\right)$, in $\Gamma\left(S_{0}\right)$, of the origin $\langle\mathrm{id}\rangle$ of $X\left(S_{0}\right)$ such that the quotient space $N / \Gamma_{0}\left(S_{0}\right)$ is a neighborhood of $\left[S_{0}\right]$ in $M(g, n)$. Hence there is a positive integer $\rho$ such that $f^{\rho}$ is homotopic to a product of $\nu$-th powers of Dehn twists about Jordan curves mapped by $\alpha$ into nodes, where $\alpha: S \rightarrow S_{0}$ is a deformation as in $\S 3$. Now we set $E=(|\zeta|<1)$ and $E^{*}=E-\{0\}$ in the $\zeta$-plane. Let $\kappa: E \rightarrow D$ be the mapping sending $\zeta$ into $\zeta^{\rho}$. We consider the holomorphic family $\left(\mathscr{S}^{\prime} \pi^{\prime}, E^{*}\right)$ 
constructed from $\left(\mathscr{S}, \pi, D^{*}\right)$ by the relation $t=\zeta^{\circ}$. Let $S_{\zeta}^{\prime}$ be the fibre of $\mathscr{S}^{\prime}$ over $\zeta$ in $E^{*}$ and let $\left[S, f_{\xi}^{\prime}, S_{\xi}^{\prime}\right]$ be a point of $T(S)$ corresponding to a point $\widetilde{\Phi}(\tau)$ of $T(G)$ for a certain $\tau$ in $\widetilde{D}$ with $\zeta^{o}=p(\tau)$. Then the analytic mapping $K: E^{*} \rightarrow X^{\prime}\left(a\left(S_{0}\right)\right)$ sending $\zeta$ into $\left\langle a\left(S_{\zeta}^{\prime}\right), a\left(\alpha \circ f_{!}^{\prime-1}\right), a\left(S_{0}\right)\right\rangle$ is single-valued. Thus $K$ has a holomorphic extension $\hat{K}: E \rightarrow X^{\prime}\left(a\left(S_{0}\right)\right)$ with $\hat{K}(0)=\langle$ id $\rangle$.

Let $H(\zeta)$ be a Kleinian group determined by the point $\hat{K}(\zeta)$ of $X^{\prime}\left(a\left(S_{0}\right)\right)$. Let $\Omega(\zeta)$ be the part of the region of discontinuity of $H(\zeta)$ corresponding to $S_{\zeta}^{\prime}$ for $\zeta$ in $E$, and let $\Omega^{\prime}(\zeta)$ be the open set obtained from $\Omega(\zeta)$ by deleting all elliptic fixed points of $H(\zeta)$. We set

$$
\mathscr{R}=\left\{(\zeta,[z]) \mid \zeta \in E^{*}, \quad[z] \in \Omega^{\prime}(\zeta) / H(\zeta)\right\}
$$

and

$$
\hat{\mathscr{\mathscr { C }}}=\left\{(\zeta,[z]) \mid \zeta \in E^{*}, \quad[z] \in \Omega(\zeta) / H(\zeta)\right\} .
$$

Then, by definition, $\mathscr{R}$ and $\hat{\mathscr{R}}$ are two dimensional complex manifolds and $\mathscr{R}$ is a Zariski open subset of $\hat{\mathscr{R}}$. If $I I: \mathscr{R} \rightarrow E^{*}$ and $\hat{\Pi}: \hat{\mathscr{R}} \rightarrow E^{*}$ are canonical projections, then $\left(\mathscr{R}, \Pi, E^{*}\right)$ is a holomorphic family of Riemann surfaces of type $(g, n)$ and $\left(\hat{\mathscr{R}}, \hat{\Pi}, E^{*}\right)$ is a holomorphic family of compact Riemann surfaces of genus $g$.

Let $\widetilde{\Psi}$ and $\widetilde{\Psi}_{1}$ be the representations of $\left(\mathscr{P}^{\prime}, \pi^{\prime}, E^{*}\right)$ and $\left(\mathscr{R}, \Pi, E^{*}\right)$ into $T(G)$ as in $\S 1.2$, respectively. For a certain positive integer $\rho=\rho_{0}$, we see that $\widetilde{\Psi}=\widetilde{\Psi}_{1}$. Hence we may assume that $\left(\mathscr{S}^{\prime}, \pi^{\prime}, E^{*}\right)$ and $\left(\mathscr{R}, \Pi, E^{*}\right)$ have the same homotopic monodromy $\mathscr{C l}_{1}$ for a certain positive integer $\rho=\rho_{0}$. So we can naturally identify $\left(\mathscr{S}^{\prime}, \pi^{\prime}, E^{*}\right)$ with $\left(\mathscr{R}, \Pi, E^{*}\right)$.

For each $\zeta$ of $E^{*}$, we set $R_{\zeta}=\Omega^{\prime}(\zeta) / H(\zeta)=\Pi^{-1}(\zeta)$ and $\hat{R}_{\zeta}=\Omega(\zeta) / H(\zeta)=$ $\hat{\Pi}^{-1}(\zeta)$. Let $R_{0}^{\prime}=\Omega^{\prime}(0) / H(0)$ and let $R_{0}$ be the union of $R_{0}^{\prime}$ and the images of all elliptic vertices of $H(0)$ corresponding to the nodes of $S_{0}$, where all related elliptic vertices are identified. Then, by the construction, $R_{0}$ is isomorphic to $S_{0}$. We also set $\hat{R}_{0}=\Omega(0) / H(0)$ with the images of all related elliptic vertices of $H(0)$ identified, that is, the compactification of $R_{0}$. Finally, we set

$$
\mathscr{R}_{0}=\hat{\mathscr{R}} \cup\left\{(0,[z]) \mid[z] \in R_{0}\right\}
$$

and

$$
\hat{\mathscr{R}}_{0}=\hat{\mathscr{R}} \cup\left\{(0,[z]) \mid[z] \in \hat{R}_{0}\right\} .
$$

Then $\mathscr{Z}_{0}$ is a two dimensional complex manifold. By the same reasoning as in the proof of Theorem 4 in [6], we can prove that $\hat{\mathscr{R}}_{0}$ has a normal complex structure such that its restriction to $\mathscr{R}_{0}$ is the same one given 
on $\mathscr{R}_{0}$ and $\hat{\mathscr{R}}_{0}-\mathscr{R}_{0}$ is a proper analytic subset of $\hat{\mathscr{R}}_{0}$. Thus the projection $\hat{\Pi}: \hat{\mathscr{R}} \rightarrow E^{*}$ has a holomorphic extension $\hat{\Pi}_{0}: \hat{\mathscr{R}}_{0} \rightarrow E$.

If we take an element $\omega_{0} \in N(G)$ with $\left\langle\omega_{0}\right\rangle=\mathscr{M}$ and set $\omega_{1}=\omega_{0}^{o_{0}}$, then $\left\langle\omega_{1}\right\rangle=\mathscr{C}_{1}$. It should be noted that the action of $\hat{I}_{\rho_{0}} \in \hat{\mathscr{G}}$ on $\tilde{\mathscr{D}}_{1}$ is trivial, where $I \in G$ is the identity and $\tilde{\mathscr{D}}_{1}$ is the one defined in $\S 4.2$. Let $\mathscr{G}_{1}$ be the subgroup of $\mathscr{G}$ generated by $\omega_{1}$ and $G$. Denote by $\hat{\mathscr{G}}_{1}$ the subgroup of $\hat{\mathscr{G}}$ induced by $\mathscr{G}_{1}$. Let $p_{1}: \widetilde{D} \rightarrow E^{*}$ be the holomorphic mapping with the relation $p(\tau)=p_{1}(\tau)^{\rho_{0}}$, where $p: \widetilde{D} \rightarrow D^{*}$ is as in $\S 1.2$.

We will canonically construct a biholomorphic mapping $\mathscr{F}: \mathscr{D} / \mathscr{G}_{1} \rightarrow$ $\mathscr{R}$. For that purpose, let $\pi_{0}: U \rightarrow S=U / G$ be the canonical projection. For a point $\tau$ of $\widetilde{D}$ and for a point $[z]$ of $D_{\tau} / G_{\tau}$, we set

$$
F_{\tau}([z])=h_{\hat{\delta}, \tau} \circ \pi_{0} \circ W_{\tau}^{-1}(z),
$$

where $h_{\hat{\delta}, \tau}$ and $W_{\tau}$ are those in $\S 4.1$. Then the mapping $F_{\tau}: D_{\tau} / G_{\tau} \rightarrow R_{p_{1}(\tau)}$ is conformal. If two points $(\tau, z)$ and $\left(\tau^{\prime}, z^{\prime}\right)$ in $\mathscr{D}$ are equivalent under $\mathscr{G}_{1}$, then $F_{\tau}([z])=F_{\tau^{\prime}}\left(\left[z^{\prime}\right]\right)$. Thus these mappings $\left\{F_{\tau}\right\}_{\left\ulcorner\in D^{*}\right.}$ induce a biholomorphic mapping $\mathscr{F}: \mathscr{D} / \mathscr{G}_{1} \rightarrow \mathscr{R}$. This mapping $\mathscr{F}$ can be extended to a homeomorphism $\hat{\mathscr{F}}: \hat{\mathscr{D}} \mid \hat{\mathscr{G}}_{1} \rightarrow \hat{\mathscr{R}}_{0}$. In fact, by the argument similar to that in the proof of Lemma 2 in [6], we can construct an analytic isomorphism $\hat{F}_{1}$ of $\left(D_{1} \cup\right.$ \{parabolic fixed points on $\partial D_{1}$ of $\left.\left.G_{1}\right\}\right) / G_{1}$ onto $\hat{R}_{0}$ by using the mappings $V_{j, \tau}=W_{\tau} \circ A_{\jmath}^{-1} \circ W_{j, \varepsilon}^{-1}$ appearing in $\S 4.1$. For a point $(1, z)$ in $\tilde{\mathscr{D}}_{1}$, we set $\hat{\mathscr{F}}([1, z])=\left(0, \hat{F}_{1}([z])\right)$. Since the action $\hat{I}_{\rho_{0}}$ on $\tilde{\mathscr{D}}_{1}$ is trivial, the mapping $\hat{\mathscr{F}}$ is well-defined and is bijective. By the definition of topologies of $\hat{\mathscr{D}} \mid \hat{\mathscr{G}}_{1}$ and of $\hat{\mathscr{R}}_{0}$ and by the construction of $\hat{\mathscr{F}}$, we can prove that $\hat{\mathscr{F}}$ is homeomorphic.

6. Let $F(G)$ be the fibre space over the Teichmüller space $T(G)$ and take an element $\langle\omega\rangle=\mathscr{l}$. For every element $[w]$ of $T(G)$, we set $w^{\prime}=$ $\lambda \circ w \circ \omega^{-1} \in Q_{\text {norm }}(G)$, where $\lambda$ is a real Möbius transformation. Let $\phi$ and $\phi^{\prime}$ be the quadratic differentials associated with $[w]$ and $\left[w^{\prime}\right]$, respectively. We set $\widehat{z}=W_{\phi}, \circ \omega \circ W_{\phi}^{-1}(z)$ for $z \in D_{\phi}$. Then $[\omega]_{*}([w], z)=\left(\left[w^{\prime}\right], \hat{z}\right)$ induces an analytic automorphism $[\omega]_{*}$ of $F(G)$. This analytic automorphism $[\omega]_{*}$ induces a finite subgroup $\Sigma$ of the analytic automorphism group of $\mathscr{D} / \mathscr{G}_{1}$ and every element $\sigma$ of $\Sigma$ can be extended to a homeomorphism $\hat{\sigma}$ of $\hat{\mathscr{D}} / \hat{\mathscr{E}}_{1}$ onto itself. We set $\hat{\Sigma}=\{\hat{\sigma} \mid \sigma \in \Sigma\}$ and $\hat{\Sigma}_{0}=\hat{\mathscr{F}} \circ \hat{\Sigma} \circ \hat{\mathscr{F}}^{-1}$. Since $\hat{\mathscr{R}}_{0}$ is a normal complex space as mentioned in $\S 4.5$, every element of $\hat{\Sigma}_{0}$ is an analytic automorphism of $\hat{\mathscr{R}}_{0}$. By Cartan's theorem, $\hat{\mathscr{R}}_{0} / \widehat{\Sigma}_{0}$ becomes a normal complex space and $\mathscr{F}$ induces an analytic isomorphism of $\mathscr{S}_{1}=\left(\mathscr{D} / \mathscr{G}_{1}\right) / \Sigma$ onto $\mathscr{R} / \hat{\Sigma}_{0}$, which can be extended to a homeomorphism 
of $\hat{\mathscr{S}}=\left(\hat{\mathscr{D}} \mid \hat{\mathscr{S}}_{1}\right) / \hat{\Sigma}$ onto $\hat{\mathscr{R}}_{0} / \hat{\Sigma}_{0}$. By this identification, $\hat{\mathscr{S}}$ has a normal complex structure. Let $\pi_{1}: \mathscr{S}_{1} \rightarrow D^{*}$ and $\hat{\pi}: \hat{\mathscr{S}} \rightarrow D$ be the natural projections, respectively. Then $\left(\mathscr{S}, \pi, D^{*}\right)$ is analytically equivalent to $\left(\mathscr{S}_{1}, \pi_{1}, D^{*}\right)$ and $(\hat{\mathscr{S}}, \hat{\pi}, D)$ is a completion of $\left(\mathscr{S}_{1}, \pi_{1}, D^{*}\right)$. Therefore, $\hat{\mathscr{S}}=\hat{\mathscr{D}} / \hat{\mathscr{G}}$ is a normal complex space and $(\hat{\mathscr{S}}, \hat{\pi}, D)$ is a completion of $\left(\mathscr{S}, \pi, D^{*}\right)$.

Summarizing the results obtained above, we have the following theorem.

THEOREM 2. For a holomorphic family $\left(\mathscr{S}, \pi, D^{*}\right)$ of Riemann surfaces of type $(g, n)$ with $2 g-2+n>0$, a completion $(\hat{\mathscr{S}}, \hat{\pi}, D)$ of $\left(\mathscr{S}, \pi, D^{*}\right)$ is canonically constructed in such a way that $(\hat{\mathscr{S}}, \hat{\pi}, D)$ is a holomorphic family of compact Riemann surfaces of genus $g$ with or without a singular fibre over $t=0$ and that $\hat{\mathscr{S}}$ is a two dimensional normal complex space.

\section{An extension theorem.}

THEOREM 3. Let $\bar{S}$ be a two dimensional complex manifold and let $\bar{\pi}$ be a proper holomorphic mapping of $\overline{\mathscr{S}}$ onto the unit disc. Assume that there is a one dimensional analytic subset $C$ of $\overline{\mathscr{S}}$ such that, setting $\mathscr{S}=\mathscr{S}-\bar{\pi}^{-1}(0) \cup C, \pi=\bar{\pi} \mid \mathscr{S}$ and $D^{*}=$ the punctured unit disc, $\pi: \mathscr{S} \rightarrow D^{*}$ is a holomorphic family of Riemann surfaces of type $(g, n)$ with $2 g-2+n>0$. Let $\left(\hat{\mathscr{S}}, \hat{\pi}, D^{*}\right)$ be the completion of $\left(\mathscr{S}, \pi, D^{*}\right)$ canonically constructed in Theorem 2. Then every holomorphic mapping $F: \mathscr{S} \rightarrow \hat{S}$ with $\pi=\hat{\pi} \circ F$ can be extended to a meromorphic mapping $\hat{F}: \overline{\mathscr{S}} \rightarrow \hat{\mathscr{S}}$.

Proof. By the construction of $\hat{\mathscr{S}}$, the argument similar to that in the proof of Lemma 1 in [6] which uses Kobayashi's extension theorem shows that $F$ can be extended to a holomorphic mapping $\widetilde{F}:(\overline{\mathscr{S}}-A) \rightarrow$ $\hat{\mathscr{S}}$, where $A$ is the set of singular points of $\bar{\pi}^{-1}(0) \cup C$. If $\hat{S}_{0}$ is the fibre of $\hat{\mathscr{S}}$ over $t=0$, then the graph $\Gamma=\{(P, \widetilde{F}(P)) \mid P \in \overline{\mathscr{S}}-A\}$ of $\widetilde{F}$ is an analytic subset of $(\overline{\mathscr{S}} \times \hat{\mathscr{S}})-\left(A \times \widehat{S}_{0}\right)$. Since $\operatorname{dim}\left(A \times \widehat{S}_{0}\right)=1$ and $\operatorname{dim}_{x}(\Gamma)=2$ for every point $x$ of $\Gamma$, Remmert-Stein's theorem implies that the closure $\bar{\Gamma}$ of $\Gamma$ in $\overline{\mathscr{S}} \times \hat{\mathscr{S}}$ is an analytic subset of $\overline{\mathscr{S}} \times \hat{\mathscr{S}}$. Further, for the canonical projections $\bar{\Pi}: \overline{\mathscr{S}} \times \hat{\mathscr{S}} \rightarrow \overline{\mathscr{S}}$ and $\hat{\Pi}: \overline{\mathscr{S}} \times$ $\hat{\mathscr{S}} \rightarrow \hat{\mathscr{S}}$, the mappings $\bar{\Pi} \mid \bar{\Gamma}$ and $\hat{\Pi} \mid \bar{\Gamma}$ are both proper holomorphic mappings. Thus $\widetilde{F}$ can be extended to a meromorphic mapping $\hat{F}: \overline{\mathscr{S}} \rightarrow$ $\hat{\mathscr{S}}$. This completes the proof of Theorem 3 . 
6. Uniformization of algebraic surfaces. 1. In the first place, we explain the uniformization theorem due to Griffiths [5] along Bers's line [1] and [4].

Let $X$ be a two dimensional irreducible non-singular projective algebraic variety over $C$ and let $X_{1}$ be a non-empty Zariski open subset of $X$. Assume that $X$ is embedded in the $N$-dimensional projective space $\boldsymbol{P}_{N}$ for some $N$. We can find two homogeneous polynomials $F_{0}$ and $F_{1}$ of the same degree in $N+1$ variables and two non-empty Zariski open subsets $Y\left(\subset X_{1}\right)$ and $Z\left(\subset \boldsymbol{P}_{1}\right)$ such that the mapping $\pi$ of $\boldsymbol{P}_{N}$ onto $\boldsymbol{P}_{1}$ sending $\zeta$ into $\left(F_{0}(\zeta), F_{1}(\zeta)\right)$ is a well-defined mapping of $Y$ onto $Z$ and is of maximal rank at all points of $Y$ and such that for every $z$ of $Z$, the fibre $S_{z}=\pi^{-1}(z) \cap Y$ of $Y$ over $z$ is a Riemann surface of (fixed) finite type $(g, n)$ with $2 g-2+n>0$. Replacing $Z$ by a smaller Zariski open subset if necessary, we may assume that the universal covering space $\widetilde{Z}$ of $Z$ is the unit disc in the complex plane. Let $p: \widetilde{Z} \rightarrow Z$ be the universal covering.

Let $G$ be a finitely generated Fuchsian group of the first kind with no elliptic elements acting on the upper half-plane $U$ such that the quotient space $S=U / G$ is of type $(g, n)$. By the same argument as in $\S 1.2$, we see that there is a holomorphic mapping $\widetilde{\Phi}: \widetilde{Z} \rightarrow T(G)$ such that $D_{\widetilde{\Phi}(\tau)} / G_{\widetilde{\Phi}_{(\tau)}}$ is conformally equivalent to $S_{p(\tau)}$ for every $\tau \in \widetilde{Z}$.

Let $\mathscr{D}$ be the set of all pairs $(\tau, w)$ with $\tau \in \widetilde{Z}$ and $w \in D_{\widetilde{\phi}_{(\tau)} \text {. Then }}$ $\mathscr{D}$ is a bounded Bergman domain in $C^{2}$. The group $G$ operates on $\mathscr{D}$ as a discrete and fixed-point-free group of analytic automorphisms by the rule

$$
g(\tau, w)=\left(\tau, W_{\widetilde{\Phi}_{(\tau)}} \circ g \circ W_{\tilde{\Phi}(\tau)}^{-1}(w)\right)
$$

for $(\tau, w) \in \mathscr{D}$ and $g \in G$, where $W_{\widetilde{\Phi}_{(\tau)}}$ is the quasiconformal automorphism of $\hat{\boldsymbol{C}}$ defined by $\widetilde{\Phi}(\tau)$ as in $\S 1.1$. The quotient space $\mathscr{D} / G$ is a two dimensional complex manifold and the canonical projection $\mathscr{D} \rightarrow \mathscr{D} / G$ is a universal covering. A point of $\mathscr{D} / G$ may be regarded as a pair $(\tau, a)$ with $\tau \in Z$ and $a \in S_{p(\tau)}$ such that $\pi(a)=p(\tau)$. Follow the canonical projection $\mathscr{D} \rightarrow \mathscr{D} / G$ by the holomorphic mapping which sends $(\tau, \alpha)$ to a. Then the composed mapping $\mathscr{D} \rightarrow Y$ is considered to be a universal covering. Hence the universal covering space $\widetilde{Y}$ of $Y$ is the bounded Bergman domain $\mathscr{D}$ in $C^{2}$. This is Griffiths's uniformization theorem of algebraic surfaces.

2. As an application of the completion of holomorphic families of Riemann surfaces of type $(g, n)$ stated in $\S 4$, we can give a supplement to the above uniformization theorem. 
Let $\Gamma$ be the covering transformation group of the universal covering $p: \widetilde{Z} \rightarrow Z$. The group $\Gamma$ acting on the unit disc $\widetilde{Z}$ is a finitely generated Fuchsian group of the first kind. Denote by $\mathscr{C}$ the set of all parabolic fixed points of $\Gamma$. For each point $\tau_{0}$ in $\mathscr{C}$, we set $\widetilde{\Phi}\left(\tau_{0}\right)=\lim _{\tau \rightarrow \tau_{0}} \widetilde{\Phi}(\tau)$, where the limit is taken in a cusp region at $\tau_{0}$ in $\widetilde{Z}$. For each $\tau \in \widetilde{Z} \cup$ $\mathscr{C}$, we set $G_{\tau}=G_{\Phi(\tau)}, \quad \Omega\left(G_{\tau}\right)=$ the region of discontinuity of $G_{\tau}, \quad \Delta\left(G_{\tau}\right)=$ the invariant component of $G_{\tau}$ corresponding to the lower half-plane, and $D_{\tau}=\Omega\left(G_{\tau}\right)-\Delta\left(G_{\tau}\right)$. We denote by $\mathscr{P}_{\tau}$ the set of all parabolic fixed points on $\partial D_{\tau}$ of $G_{\tau}$ for every $\tau \in \widetilde{Z} \cup \mathscr{C}$. We set

$$
\hat{\mathscr{D}}=\left\{(\tau, w) \mid \tau \in \widetilde{Z} \cup \mathscr{C}, \quad w \in D_{\tau} \cup \mathscr{P}_{\tau}\right\} .
$$

Each point of $\hat{\mathscr{D}}-\mathscr{D}$ is called a cusp point of $\mathscr{D}$. We can canonically introduce a Hausdorff topology on $\hat{\mathscr{D}}$ as in $\S 4.2$.

3. As was stated in $\S 4.3$, every element of $\Gamma$ or $G$ induces an analytic automorphism of $\mathscr{D}$, which can be extended to a topological automorphism of $\hat{\mathscr{D}}$. Denote by $\mathscr{G}$ the discrete and fixed-point-free group of all such analytic automorphisms of $\mathscr{D}$ induced by $\Gamma$ and $G$, and denote by $\hat{\mathscr{G}}$ the group of all topological automorphisms of $\hat{\mathscr{D}}$ induced by $\mathscr{G}$. The quotient space $\mathscr{S}=\mathscr{D} / \mathscr{G}$ is a two dimensional manifold and $\mathscr{S}$ is biholomorphically equivalent to $Y$. By this identification of $\mathscr{S}$ with $Y$, the canonical projection $\mathscr{D} \rightarrow \mathscr{S}$ is the universal covering of $Y$ and its covering transformation group is $\mathscr{G}$. By Theorem 2 , the quotient space $\hat{\mathscr{S}}=\hat{\mathscr{D}} / \hat{\mathscr{G}}$ is a two dimensional compact normal complex space. Further, by Theorem $3, X$ is bimeromorphically equivalent to $\hat{\mathscr{P}}$.

Thus we have the following theorem.

THEOREM 4. Let $X$ be a two dimensional, irreducible, non-singular projective algebraic variety over $C$ and let $X_{1}$ be a non-empty Zariski open subset of $X$. Then there is a non-empty Zariski open subset $Y$ of $X_{1}$ such that the universal covering space $\mathscr{D}$ of $Y$ can be canonically constructed and is a bounded Bergman domain in $C^{2}$. Moreover, if $\hat{\mathscr{D}}$ is the union of $\mathscr{D}$ and all its cusp points and if $\mathscr{G}$ is its covering transformation group, then $\hat{\mathscr{D}}$ has a natural Hausdorff topology and every element of $\mathscr{G}$ can be extended to a topological automorphism of $\hat{\mathscr{D}}$. If $\hat{\mathscr{G}}$ is the group of all topological automorphisms of $\hat{\mathscr{D}}$ induced by $\mathscr{G}$, then the quotient space $\hat{\mathscr{S}}=\hat{\mathscr{D}} \mid \hat{\mathscr{G}}$ is a two dimensional compact normal complex space and is bimeromorphically equivalent to $X$.

7. Compactification of two dimensional Stein manifolds with holomorphic fibration. 1. We consider the compactification of a two dimen- 
sional Stein manifold with a certain holomorphic fibration. Let $M$ be a compact analytic space and $T$ be an analytic subset of $M$. We call $M$ a compactification of a complex manifold $X$ if $M-T$ and $X$ are biholomorphically equivalent.

We can prove the following.

THeorem 5. Let $\mathscr{S}$ be a two dimensional Stein manifold, let $R_{0}$ be a compact Riemann surface and let $R$ be a non-empty Zariski open subset of $R_{0}$. Assume that there exists a holomorphic mapping $\pi: \mathscr{S} \rightarrow R$ such that

1) $\pi$ is of maximal rank at every point of $\mathscr{S}$, and

2) for every point $t$ of $R$, the fibre $S_{t}=\pi^{-1}(t)$ of $\mathscr{S}$ over $t$ is an irreducible analytic subset of $\mathscr{S}$ and is of fixed finite type $(g, n)$ with $2 g-2+n>0$ as a Riemann surface.

Then a compactification $\hat{\mathscr{S}}$ of $\mathscr{S}$ can be canonically constructed and $\hat{\mathscr{S}}$ is normal and is bimeromorphically equivalent to a projective algebraic surface. Moreover, every compactification of $\mathscr{S}$ is bimeromorphically equivalent to $\hat{\mathscr{S}}$.

Proof. We can construct a two dimensional complex manifold $\overline{\mathscr{S}}$ such that $\mathscr{S}$ can be regarded as a Zariski open subset of $\mathscr{S}$ and that $C=\overline{\mathscr{S}}-\mathscr{S}$ is a non-singular one dimensional analytic subset. (See Theorem II in Nishino [10].) The mapping $\pi: \mathscr{S} \rightarrow R$ can be extended to a proper holomorphic mapping $\bar{\pi}: \overline{\mathscr{S}} \rightarrow R$. Hence Theorem 2 implies that a completion $\left(\hat{\mathscr{S}}, \hat{\pi}, R_{0}\right)$ of $(\mathscr{S}, \pi, R)$ can be canonically constructed and $\hat{\mathscr{S}}$ is a two dimensional compact normal complex space. This space $\hat{\mathscr{S}}$ is a compactification of $\mathscr{S}$.

In order to prove that $\hat{\mathscr{S}}$ is bimeromorphically equivalent to a projective algebraic surface, it is sufficient to show that the algebraic dimension $a(\hat{\mathscr{S}})$ of $\hat{\mathscr{S}}$ is equal to 2. (See Theorem 3.1 in Kodaira [8].) In our case, obviously $a(\hat{\mathscr{S}})=1$ or 2 . The set $C=\overline{\mathscr{S}}-\mathscr{S}$ can be regarded as a one dimensional non-singular analytic subset $\mathscr{C}$ of $\hat{\mathscr{S}}_{0}=$ $\hat{\mathscr{S}}-\hat{\pi}^{-1}\left(R_{0}-R\right)$. By the same reasoning as in the proof of Theorem 3 , we can prove that $\mathscr{C}$ has an analytic extension $\hat{\mathscr{C}}$, that is, $\hat{\mathscr{C}}$ is an analytic subset of $\hat{\mathscr{S}}$ with $\mathscr{C}=\hat{\mathscr{C}} \cap \hat{\mathscr{S}}_{0}$. Thus the one dimensional compact analytic subset $\hat{\mathscr{C}}$ intersects every fibre $\hat{S}_{t}=\hat{\pi}^{-1}(t)$ of $\hat{\mathscr{S}}$. Therefore, Kodaira's theorem implies that $a(\hat{\mathscr{S}})$ is not equal to 1 . (See Theorem 4.3 in Kodaira [9].) So we have $a(\hat{\mathscr{S}})=2$ and we see that $\hat{\mathscr{S}}$ is bimeromorphically equivalent to a projective algebraic surface. 
For a compactification $M$ of $\mathscr{S}$ with the inclusion map $j^{\prime}$, we set $\mathscr{S}^{\prime}=j^{\prime}(\mathscr{S})$ and $\pi^{\prime}=\pi \circ j^{\prime-1}$. The triple $\left(\mathscr{S}^{\prime}, \pi^{\prime}, R\right)$ is a holomorphic family of Riemann surfaces of type $(g, n)$. Let $j$ be the inclusion map of $\mathscr{S}$ into $\hat{\mathscr{S}}$ and let $J=j \circ j^{\prime-1}$. Denote by $A$ the one dimensional analytic subset $M-\mathscr{S}^{\prime}$ of $M$. In the same manner as in the proof of Theorem 3 , we can prove that $J$ can be extended to a holomorphic mapping $\widetilde{J}: M-\operatorname{Sing}(A) \rightarrow \hat{\mathscr{S}}$, where $\operatorname{Sing}(A)$ is the set of singular points of $A$. By the relation $\pi^{\prime}=\hat{\pi} \circ J$ on $\mathscr{S}^{\prime}$, the mapping $\pi^{\prime}: \mathscr{S}^{\prime} \rightarrow R$ can be extended to a holomorphic mapping $\Pi: M-\operatorname{Sing}(A) \rightarrow R_{0}$. Since the codimension of $\operatorname{Sing}(A)$ is not less than 2 and since the compact Riemann surface $R_{0}$ is a projective algebraic curve, Levi's extension theorem implies that $\Pi$ can be extended to a meromorphic mapping of $M$ onto $R_{0}$. There exists a finite succession $\sigma: \widetilde{M} \rightarrow M$ of $\sigma$-processes centered at the points of $\operatorname{Sing}(A)$ such that $\widetilde{\Pi}=\Pi \circ \sigma$ of $\widetilde{M}$ onto $R_{0}$ is a proper holomorphic mapping. Hence we have a holomorphic family $\left(\sigma^{-1}\left(\mathscr{S}^{\prime}\right)\right.$, $\widetilde{\Pi}, R)$ of Riemann surfaces of type $(g, n)$. Theorem 3 implies that the holomorphic mapping $J \circ \sigma$ of $\sigma^{-1}\left(\mathscr{S}^{\prime}\right)$ into $\hat{\mathscr{S}}$ with the relation $\widetilde{\Pi}=$ $\hat{\pi} \circ(J \circ \sigma)$ on $\sigma^{-1}\left(\mathscr{S}^{\prime}\right)$ can be extended to a bimeromorphic mapping of $\tilde{M}$ onto $\hat{\mathscr{S}}$. Since $M$ is bimeromorphically equivalent to $\widetilde{M}$, we see that $M$ is also bimeromorphically equivalent to $\hat{\mathscr{S}}$. This completes the proof of Theorem 5.

REMARK. If $2 g-2+n \leqq 0$, then there is a two dimensional Stein manifold $\mathscr{S}$ with a holomorphic fibration $(\mathscr{S}, \pi, R)$ of type $(g, n)$ such that a compactification $\hat{\mathscr{S}}$ of $\mathscr{S}$ is not bimeromorphically equivalent to a projective algebraic surface. We shall give an example. Let $T$ be a linear automorphism of $C^{2}$ sending $(z, w)$ into $\{(1 / 2)(z+w),(1 / 2) w\}$ and let $G$ be the group generated by $T$. Since $G$ is a properly discontinuous group with no fixed points in $C^{2}-\{0\}$, the quotient space $\hat{\mathscr{S}}=\left(C^{2}-\{0\}\right) / G$ is a two dimensional compact complex manifold. Such a surface $\hat{\mathscr{S}}$ is called a Hopf surface. Since $\hat{\mathscr{S}}$ is diffeomorphic to $S^{1} \times S^{3}$, the first Betti number is odd and is equal to 1 , which implies that $\hat{\mathscr{S}}$ is not a Kähler manifold. Thus $\hat{\mathscr{S}}$ is not algebraic. Moreover, we can prove that there is no meromorphic functions on $\hat{\mathscr{S}}$ other than constant functions. We can also prove that there is no one dimensional analytic subset of $\hat{\mathscr{\rho}}$ except for a non-singular elliptic curve $C=\{(z, 0) \mid z \in C-$ $\{0\}\} / G$. If we set $\mathscr{S}=\hat{\mathscr{S}}-C$ and $\Pi([z],[w])=\exp (2 \pi i z / w)$ for $(z, w)$ of $C^{2}-\{w=0\}$, then $\mathscr{S}$ is biholomorphically equivalent to $\boldsymbol{C}^{*} \times \boldsymbol{C}^{*}$ by the mapping sending $[z, w]$ into $\{\exp (2 \pi i z / w), w \exp ((z / w) \log 2)\}$ and the 
triple $\left(\mathscr{S}, \Pi, C^{*}\right)$ is a holomorphic family of Riemann surfaces of type $(0,2)$, where $C^{*}=C-\{0\}$.

Finally, as an application of Theorems 3 and 5, we will prove the following Theorem 6 which is due to Kizuka [7].

Let $P(x, y)$ be a non-constant polynomial of two complex variables $x$ and $y$. For any complex number $c$, each irreducible component $S_{c}$ of the analytic subset $\left\{(x, y) \in C^{2} \mid P(x, y)=c\right\}$ of $C^{2}$ is called a prime surface of $P$ with value $c$. If $\widetilde{S}_{c}$ is the desingularization of $S_{c}$ and if $\widetilde{S}_{c}$ is of type $(g, n)$ as a Riemann surface, we say that $S_{c}$ is of type $(g, n)$. For all values of $c$ except for a finite number of values, every prime surface $S_{c}$ of $P$ is non-singular and is of fixed finite type $\left(g_{0}, n_{0}\right)$. If $2 g_{0}-2+$ $n_{0}>0$, then the polynomial $P$ is said to be of general type.

THEOREM 6. Let $T$ be an analytic automorphism of $C^{2}$. If there exists a polynomial $P$ of general type such that $P \circ T$ is also a polynomial, then $T$ is a polynomial map.

Proof. For the polynomial $P$, there exists a polynomial $P_{0}(x, y)$ and a polynomial $\phi(z)$ of a complex variable $z$ such that $P(x, y)=\phi\left(P_{0}(x, y)\right)$ and that, for all values except for a finite number of values, the analytic subset $\left\{(x, y) \mid P_{0}(x, y)=c\right\}$ of $C^{2}$ is non-singular, irreducible and of order 1. So we may assume that the analytic subset $\{(x, y) \mid P(x, y)=c\}$ is non-singular, irreducible and of order 1 for all values except for a finite number of values.

We set $Q=P \circ T$. There are two one dimensional analytic subsets $C_{1}$ and $C_{2}$ of the two dimensional complex projective space $\boldsymbol{P}_{2}$ such that, if we set $\mathscr{S}_{1}=\boldsymbol{P}_{2}-C_{1}, \mathscr{S}_{2}=\boldsymbol{P}_{2}-C_{2}, \pi_{1}=P\left|\mathscr{S}_{1}, \pi_{2}=Q\right| \mathscr{S}_{2}$ and $R=\mathrm{a}$ Zariski open subset of $\boldsymbol{P}_{1}$, then $\left(\mathscr{S}_{1}, \pi_{1}, R\right)$ and $\left(\mathscr{S}_{2}, \pi_{2}, R\right)$ are holomorphic families of Riemann surfaces of type $\left(g_{0}, n_{0}\right)$ with $2 g_{0}-2+n_{0}>0$. The analytic automorphism $T$ of $C^{2}$ induces a biholomorphic mapping $T_{0}$ of $\mathscr{S}_{1}$ onto $\mathscr{S}_{2}$ with $\pi_{1}=\pi_{2} \circ T_{0}$.

Let $\left(\hat{\mathscr{S}}_{1}, \hat{\pi}_{1}, \boldsymbol{P}_{1}\right)$ and $\left(\hat{\mathscr{S}}_{2}, \hat{\pi}_{2}, \boldsymbol{P}_{1}\right)$ be the completions of $\left(\mathscr{S}_{1}, \pi_{1}, R\right)$ and $\left(\mathscr{P}_{2}, \pi_{2}, R\right)$ constructed canonically in Theorem 5 , respectively. If $j_{1}: \mathscr{S}_{1} \rightarrow$ $\hat{\mathscr{S}}_{1}$ and $j_{2}: \mathscr{S}_{2} \rightarrow \hat{\mathscr{S}}_{2}$ are the inclusion mappings, then Theorem 3 implies that $j_{1}$ and $j_{2}$ have bimeromorphic extensions $J_{1}: \boldsymbol{P}_{2} \rightarrow \hat{\mathscr{S}}_{1}$ and $J_{2}: \boldsymbol{P}_{2} \rightarrow$ $\hat{\mathscr{S}}_{2}$, respectively. Similarly, the biholomorphic mapping $j_{2} \circ T_{0} \circ j_{1}^{-1}: j_{1}\left(\mathscr{S}_{1}\right) \rightarrow$ $j_{2}\left(\mathscr{S}_{2}\right)$ has a bimeromorphic extension of $\hat{\mathscr{S}}_{1}$ onto $\hat{\mathscr{S}}_{2}$. Thus the biholomorphic mapping $T: \boldsymbol{C}^{2} \rightarrow \boldsymbol{C}^{2}$ has a bimeromorphic extension $\hat{T}: \boldsymbol{P}_{2} \rightarrow \boldsymbol{P}_{2}$, which implies that $\hat{T}$ is a rational map. Since $\hat{T} \mid C^{2}$ is holomorphic, $T$ is a polynomial map. This completes the proof of Theorem 6 . 


\section{REFERENCES}

[1] L. Bers, Uniformization, moduli and Kleinian groups, Bull. of London Math. Soc. 4 (1972), 257-300.

[2] L. BERs, Spaces of degenerating Riemann surfaces, in "Discontinuous groups and Riemann surfaces”, Ann. of Math. Studies 79, Princeton Univ. Press, (1974), 43-55.

[3] L. Bers, Deformations and moduli of Riemann surfaces with nodes and signatures, Math. Scand. 36 (1975), 12-16.

[4] L. Bers, On Hilbert's 22nd problem, Proceedings of Symposia in pure Mathematics Volume 28 (1976), 559-609.

[5] P. A. Griffiths, Complex analytic properties of certain Zariski open sets on algebraic varieties, Ann. of Math. 94 (1971), 21-55.

[6] Y. Imayoshi, Holomorphic families of Riemann surfaces and Teichmüller spaces, to appear in the Proceedings of the 1978 conference on Riemann surfaces at Stony Brook.

[7] T. Kizuka, Analytic automorphisms and algebraic automorphisms of $C^{2}$, Tôhoku Math. J. 31 (1979), this issue, 553-565.

[8] K. Kodaira, On compact analytic surfaces I, Ann. of Math. 71 (1960), 111-152.

[9] K. KodaIra, On the structure of compact analytic surfaces II, Amer. J. of Math. 88 (1966), 682-721.

[10] T. Nishino, Nouvelles recherches sur les fonctions entières de plusieurs variables complexes IV, types de surfaces premières, J. Math. Kyoto Univ. 13 (1973), 217-272.

Mathematical INSTitute

TôHOKU UNIVERSITY

SendaI, 980 Japan 
